Pacific Journal of Mathematics

LEVEL SET MAXIMA AND QUASILINEAR ELLIPTIC 


\section{LEVEL SET MAXIMA \\ AND QUASILINEAR ELLIPTIC PROBLEMS}

\section{KYRIL TINTAREV}

The present paper studies existence of solutions to the problem $\rho A^{\prime}(x)=B^{\prime}(x)$ where $A$ and $B$ are Fréchet differentiable functionals on a Banach space. For every given value of $A(x)=t$ we prove existence of a solution $x$ and present an expression for the eigenvalue $\rho=\rho(t)$. The result is applied to quasilinear elliptic equations.

1. Introduction. A typical problem of the second order studied below is

$$
-\operatorname{div} \mathscr{A}_{\xi}^{\prime}(x, \nabla u)=f(x, u),\left.\quad u\right|_{\partial \Omega}=0,
$$

where $\Omega \subset \mathbf{R}^{n}$ is an open bounded domain, $\mathscr{A}(x, \xi): \Omega \times \mathbf{R}^{n} \rightarrow \mathbf{R}$ and "ellipticity" of $\mathscr{A}$ is defined as:

$$
\begin{gathered}
\mathscr{A}(x, \cdot) \text { is convex for any } x \in \Omega, \\
\mathscr{A}_{\xi}^{\prime}(x, \xi) \cdot \xi \geq c|\xi|^{p}, \quad p>1, c>0,(x, \xi) \in \Omega \times \mathbf{R}^{n} .
\end{gathered}
$$

When $\mathscr{A}$ is a quadratic form of $\xi$ and $p=2,(1.1)$ becomes a semilinear equation. The study extends also to equations of higher order and to systems.

Our approach follows the approach of Browder [2] and Berger [1] with a subsequent refinement due to [3] and [4]. Let $X$ be a Banach space, $A, B \in C^{1}(X \rightarrow \mathbf{R})$. We consider a critical point equation

$$
\rho A^{\prime}(x)=B^{\prime}(x) \text {. }
$$

This equation might be associated with the maximum problem

$$
\sigma(t)=\sup _{A(x)=t} B(x) .
$$

Under general compactness conditions, the maximum in (1.4) is attained and provides (1.3). Remarkably the eigenvalues $\rho$ are derivatives of the function of critical values $\sigma(t)$. More precisely, since we prove that $\sigma$ might have different right and left hand derivatives $\sigma_{ \pm}^{\prime}(t)$, both of them are eigenvalues. Under additional conditions we prove that $(1.3)$ is solvable for any $\rho$ between $\inf _{t} \sigma_{-}^{\prime}(t)$ and 
$\sup _{t} \sigma_{+}^{\prime}(t)$. Thus, if the graph of $\sigma(t)$ has a slope less than 1 and a slope greater than 1 , then $A^{\prime}(u)=B^{\prime}(u)$ will be solvable. Applications to quasilinear elliptic problems then follow.

2. Maxima on the level sets. Let $X$ be a reflexive Banach space and let $A, B \in C^{1}(X \rightarrow \mathbf{R})$. Let us list several conditions to be used later. Let

$$
\begin{aligned}
S_{t} & =\{u \in X: A(u)=t\}, \\
\omega_{t} & =\{u \in X: A(u) \leq t\}
\end{aligned}
$$

and define for those $t$ when $S_{t} \neq \varnothing$,

$$
\begin{gathered}
\sigma(t)=\sup _{u \in S_{t}} B(u), \\
\beta(t)=\sup _{u \in \omega_{t}} B(u)=\sup _{\tau \leq t} \sigma(\tau) .
\end{gathered}
$$

LEMMA 2.1. Assume the following conditions:

(A1) $A$ is coercive, i.e., $\left\|u_{j}\right\| \rightarrow \infty \Rightarrow A\left(u_{j}\right) \rightarrow \infty$.

(A2) $A$ is weakly lower semicontinuous.

(A3) $\left\langle A^{\prime}(u), u\right\rangle>0$ for $u \in X \backslash\{0\}$.

(B1) $B$ has no local maxima on $X$.

(B2) $B$ is weakly continuous.

Then

$$
\begin{aligned}
& \sigma(t) \text { is increasing on }[0, \infty), \\
& \sigma(t) \text { is continuous on }[0, \infty),
\end{aligned}
$$

(2.7) the maximum in (2.3) is attained for every $t \geq 0$.

(An immediate consequence of $(2.5)$ is that $\sigma(t)=\beta(t)$.) Without loss of generality one can assume from now that

$$
A(0)=0 \text {. }
$$

Then, due to (A3)

$$
\begin{aligned}
A(u) & =\int_{0}^{1} \frac{d}{d t} A(t u) d t=\int_{0}^{1}\left\langle A^{\prime}(t u), u\right\rangle d t \\
& =\int_{0}^{1} t^{-1}\left\langle A^{\prime}(t u), t u\right\rangle d t>0, \quad \text { unless } u=0 .
\end{aligned}
$$

By (A1) the range of $A$ is $[0, \infty)$. 
Proof of Lemma 2.1. Consider a maximizing sequence $u_{j} \in \omega_{t}$ in (2.4). By (A1) it is bounded in norm. Then there is a weakly convergent renamed subsequence $u_{j} \stackrel{w}{\longrightarrow} u_{0}$. By (A2) $u_{0} \in \omega_{t}$. By (B2) $B\left(u_{j}\right) \rightarrow B\left(u_{0}\right)=\beta(t)$. If $u_{0} \notin S_{t}$, then it is a point of interior maximum for $B$ in $\omega_{t}$. This contradicts (B1). Consequently, $u_{0} \in S_{t}$. This implies in turn that $\sigma(t)=\beta(t)$ and it is a monotone increasing function. For the given $t$, the point $u_{0}$ is a point of maximum over $S_{t}$. Thus, (2.5) and (2.7) are proved.

Consider

$$
\begin{aligned}
& \sigma\left(t_{0}+0\right)=\liminf _{t \rightarrow t_{0}, t>t_{0}} \sigma(t), \\
& \sigma\left(t_{0}-0\right)=\limsup _{t \rightarrow t_{0}, t<t_{0}} \sigma(t) .
\end{aligned}
$$

Let $t_{j}>t_{0}, t_{j} \rightarrow t_{0}$ and let $\sigma\left(t_{j}\right)$ be attained an $u_{j} \in S_{t_{j}}$. Then by (A1) $u_{j}$ has a renamed weakly convergent subsequence: $u_{j} \stackrel{w}{\longrightarrow} u_{0} \in$ $\omega_{t_{0}}$. Therefore,

$$
\sigma\left(t_{0}+0\right)=B\left(u_{0}\right) \leq \sup _{u \in \omega_{t_{0}}} B(u)=\sigma\left(t_{0}\right) .
$$

The converse inequality is true by monotonicity of $\sigma$. Thus

$$
\sigma\left(t_{0}+0\right)=\sigma\left(t_{0}\right) \text {. }
$$

Let now $u_{0}$ be a point of maximum in (2.1) at $t=t_{0}$. Then by (A3)

$$
t(s):=A\left(s u_{0}\right)
$$

is a monotone increasing function and

$$
t(s) \rightarrow t_{0}, \quad t(s)<t_{0} \quad \text { as } s \rightarrow 1, s<1 .
$$

Therefore,

$$
\sigma\left(t_{0}-0\right) \geq \limsup _{\substack{s \rightarrow 1 \\ s<1}} B\left(s u_{0}\right)=\sigma\left(t_{0}\right) .
$$

The converse inequality is due to monotonicity of $\sigma$. Thus $\sigma\left(t_{0}-0\right)=$ $\sigma\left(t_{0}\right)$ and (2.6) is proved.

LEMMA 2.2. Assume the conditions of Lemma 2.1. Let $u_{0}$ be a point of maximum in (2.3), $t>0$. Then there is a $\rho \geq 0$, such that

$$
\rho A^{\prime}\left(u_{0}\right)=B^{\prime}\left(u_{0}\right) \text {. }
$$

Proof. Let $v \in X$ be such that

$$
\left\langle A^{\prime}\left(u_{0}\right), v\right\rangle<0 \text {. }
$$


By Lemma $2.1 u_{0}$ is a point of maximum of $B$ in $\omega_{t}$ and

$$
B\left(u_{0}+\theta v\right) \leq B\left(u_{0}\right)
$$

for $\theta>0$ sufficiently small. Thus,

$$
\left\langle B^{\prime}\left(u_{0}\right), v\right\rangle \leq 0
$$

for any $v$ satisfying (2.17). From (2.17), (2.19) a routine argument shows that $A^{\prime}\left(u_{0}\right)$ and $B^{\prime}\left(u_{0}\right)$ are parallel. By $(\mathrm{A} 3), A^{\prime}\left(u_{0}\right) \neq 0$ and (2.16) follows immediately.

Let us define a set $\Lambda_{t} \subset S_{t} \times[0, \infty), t \in(0, \infty)$ :

$$
\Lambda_{t}=\left\{(u, \rho): B(u)=\sigma(t), \rho A^{\prime}(u)=B^{\prime}(u)\right\} .
$$

By Lemmas 2.1, $2.2 \Lambda_{t}$ is nonempty. Let

$$
\begin{aligned}
& \Lambda_{t}^{(\rho)}=\left\{\rho \geq 0: \exists u \in S_{t},(u, \rho) \in \Lambda_{t}\right\}, \\
& \Lambda_{t}^{(u)}=\left\{u \in S_{t}: \exists \rho \geq 0,(u, \rho) \in \Lambda_{t}\right\} .
\end{aligned}
$$

By (2.20), one has also:

$$
\Lambda_{t}^{(u)}=\left\{u \in S_{t}: B(u)=\sigma(t)\right\}
$$

and

$$
\Lambda_{t}^{(\rho)}=\left\{\rho=\left\langle B^{\prime}(u), u\right\rangle /\left\langle A^{\prime}(u), u\right\rangle, u \in \Lambda_{t}^{(u)}\right\} .
$$

LEMMA 2.3. Assume the conditions of Lemma 2.1 and in addition (A4) If $u_{j} \stackrel{w}{\longrightarrow} u_{0}$, then

$$
A\left(u_{j}\right) \rightarrow A\left(u_{0}\right) \Leftrightarrow\left\langle A^{\prime}\left(u_{j}\right), u_{j}\right\rangle \rightarrow\left\langle A^{\prime}\left(u_{0}\right), u_{0}\right\rangle .
$$

(B3) $\left\langle B^{\prime}(u), u\right\rangle$ is weakly continuous.

Let $T \subset(0, \infty)$ and

$$
\Lambda_{T}=\bigcup_{t \in T} \Lambda_{t}
$$

If $T$ is compact in $(0, \infty)$, then $\Lambda_{T}$ is weakly compact in $X \times(0, \infty)$.

Proof. Let $\left(u_{j}, \rho_{j}\right) \in \Lambda_{t_{j}}, t_{j} \in T$. Consider a renamed convergent subsequence $t_{j} \rightarrow t_{0} \in T$. Then by (A1), (A2) $u_{j}$ has a weakly convergent (renamed) subsequence $u_{j} \stackrel{w}{\longrightarrow} u_{0} \in \omega_{t_{0}}$. By (B2), $B\left(u_{j}\right) \rightarrow B\left(u_{0}\right)$, by Lemma $2.1 B\left(u_{0}\right)=\sigma\left(t_{0}\right)$ and $u_{0} \in S_{t_{0}}$, i.e., 
$u_{0} \in \Lambda_{t_{0}}^{(u)}$. By Lemma $2.2 u_{j}$ satisfy (2.16) with eigenvalues, say, $\rho_{j}$, $u_{0}$ satisfies $(2.16)$ with some $\rho_{0}$, and

$$
\begin{aligned}
& \rho_{j}=\left\langle B^{\prime}\left(u_{j}\right), u_{j}\right\rangle /\left\langle A^{\prime}\left(u_{i}\right), u_{j}\right\rangle, \\
& \rho_{0}=\left\langle B^{\prime}\left(u_{0}\right), u_{0}\right\rangle /\left\langle A^{\prime}\left(u_{0}\right), u_{0}\right\rangle .
\end{aligned}
$$

Note now that $\left\langle B^{\prime}\left(u_{j}\right), u_{j}\right\rangle \rightarrow\left\langle B^{\prime}\left(u_{0}\right), u_{0}\right\rangle$ by (B3) and $\left\langle A^{\prime}\left(u_{j}\right), u_{j}\right\rangle \rightarrow$ $\left\langle A^{\prime}\left(u_{0}\right), u_{0}\right\rangle$ by (A4). Thus $\rho_{j} \rightarrow \rho_{0}$.

\section{Critical values and eigenvalues.}

THEOREM 3.1. Assume

(A1) $A$ is coercive, i.e., $\left\|u_{j}\right\| \rightarrow \infty \Rightarrow A\left(u_{j}\right) \rightarrow \infty$.

(A2) $A$ is weakly lower semicontinuous.

(A3) $\left\langle A^{\prime}(u), u\right\rangle>0$ for $u \in X \backslash\{0\}$.

(A4) If $u_{j} \stackrel{w}{\longrightarrow} u_{0}$, then

$$
A\left(u_{j}\right) \rightarrow A\left(u_{0}\right) \Leftrightarrow\left\langle A^{\prime}\left(u_{j}\right), u_{j}\right\rangle \rightarrow\left\langle A^{\prime}\left(u_{0}\right), u_{0}\right\rangle
$$

(B1) $B$ has no local maxima on $X$.

(B2) $B$ is weakly continuous.

(B3) $\left\langle B^{\prime}(u), u\right\rangle$ is weakly continuous.

Then for every $t>0$ there exist left and right derivatives $\sigma_{ \pm}^{\prime}(t), \sigma_{-}^{\prime}(t) \leq$ $\sigma_{t}^{\prime}(t)$. Moreover,

$$
\begin{aligned}
& \sigma_{+}^{\prime}(t)=\sup \left\{\rho \in \Lambda_{t}^{(\rho)}\right\} \\
& \sigma_{-}^{\prime}(t)=\inf \left\{\rho \in \Lambda_{t}^{(\rho)}\right\}
\end{aligned}
$$

Before we prove the theorem, we wish to note that supremum in (3.1) and infimum in (3.2) are attained on some $u_{ \pm} \in \Lambda_{t}^{(u)}$ due to Lemma 2.3. As a result one has

THEOREM 3.2. Under conditions of Theorem 3.2 for every $t>0$ there exist $u_{ \pm} \in S_{t}$, such that

$$
\begin{aligned}
& \sigma_{+}^{\prime}(t) A^{\prime}\left(u_{+}\right)=B^{\prime}\left(u_{+}\right), \\
& \sigma_{-}^{\prime}(t) A^{\prime}\left(u_{-}\right)=B^{\prime}\left(u_{-}\right)
\end{aligned}
$$

Proof of Theorem 3.1. 1. Let $u_{0} \in \Lambda_{t_{0}}^{(u)}$. Let $\theta_{j} \rightarrow 1, t_{j}=A\left(\theta_{j} u_{0}\right)$. Then by continuity of $A, t_{j} \rightarrow A\left(u_{0}\right)=t_{0}$. Moreover,

$$
t_{j}-t_{0}=A\left(\theta_{j} u_{0}\right)-A\left(u_{0}\right)=\left\langle A^{\prime}\left(u_{0}\right), u_{0}\right\rangle\left(\theta_{j}-1\right)+o\left(\theta_{j}-1\right) .
$$


Consequently,

$$
\begin{aligned}
\sigma\left(t_{j}\right) & -\sigma\left(t_{0}\right) \geq B\left(\theta_{j} u_{0}\right)-B\left(u_{0}\right) \\
& =\left\langle B^{\prime}\left(u_{0}\right), u_{0}\right\rangle\left(\theta_{j}-1\right)+o\left(\theta_{j}-1\right) \\
& =\left(\left\langle B^{\prime}\left(u_{0}\right), u_{0}\right\rangle /\left\langle A^{\prime}\left(u_{0}\right), u_{0}\right\rangle\right)\left(t_{j}-t_{0}\right)+o\left(t_{j}-t_{0}\right) .
\end{aligned}
$$

Fro (3.6) and (2.23) one has immediately,

$$
\begin{aligned}
& D_{+} \sigma\left(t_{0}\right) \geq \sup \left\{\rho \in \Lambda_{t_{0}}^{(\rho)}\right\}, \\
& D^{-} \sigma\left(t_{0}\right) \leq \inf \left\{\rho \in \Lambda_{t_{0}}^{(\rho)}\right\} .
\end{aligned}
$$

2. Let now $u_{j} \in \Lambda_{t_{j}}^{(u)}, t_{i} \rightarrow t_{0}$. By Lemma 2.3 a renamed sequence $u_{j}$ converges weakly to $u_{0} \in \Lambda_{t_{0}}^{(u)}$. Let us define $\theta_{j}>0$ by

$$
A\left(\theta_{j} u_{j}\right)=t_{0} .
$$

By (A3), the function $\theta \rightarrow A\left(\theta u_{j}\right)$ is monotone for any $u \neq 0$ and by (A1) the range of it is $[0, \infty)$. Thus, for given $t_{0}>0$ and $u_{j} \neq 0$, (3.9) has a unique solution $\theta_{j}>0$. Since $A \in C^{1}$, there exist $\eta_{j} \in$ $\left[\theta_{j}, 1\right]$, such that

$$
t_{j}-t_{0}=A\left(u_{j}\right)-A\left(\theta_{j} u_{j}\right)=\left\langle A^{\prime}\left(\eta_{j} u_{j}\right), u_{j}\right\rangle\left(1-\theta_{j}\right) .
$$

From (3.9) it follows that $\theta_{j}$ is a bounded sequence. Let us consider a renamed convergent subsequence: $\theta_{j} \rightarrow \theta_{0}$. Then $\theta_{j} u_{j} \stackrel{w}{\longrightarrow} \theta_{0} u_{0}$ and, necessarily, $\theta_{0}=1$. Therefore $\eta_{j} \rightarrow 1$ and

$$
t_{j}-t_{0}=\left\langle A^{\prime}\left(u_{j}\right), u_{j}\right\rangle\left(1-\theta_{j}\right)+o\left(1-\theta_{j}\right) \text {. }
$$

Similarly,

$$
B\left(u_{j}\right)-B\left(\theta_{j} u_{j}\right)=\left\langle B^{\prime}\left(u_{j}\right), u_{j}\right\rangle\left(1-\theta_{j}\right)+o\left(1-\theta_{j}\right) .
$$

Therefore

$$
\begin{aligned}
\sigma\left(t_{j}\right) & -\sigma\left(t_{0}\right) \leq\left\langle B^{\prime}\left(u_{j}\right), u_{j}\right\rangle\left(1-\theta_{j}\right)+o\left(1-\theta_{j}\right) \\
& =\left(\left\langle B^{\prime}\left(u_{j}\right), u_{j}\right\rangle /\left\langle A^{\prime}\left(u_{j}\right), u_{j}\right\rangle\right)\left(t_{j}-t_{0}\right)+o\left(t_{j}-t_{0}\right) .
\end{aligned}
$$

We have to note only that $\left\langle A^{\prime}\left(u_{j}\right), u_{j}\right\rangle \rightarrow\left\langle A^{\prime}\left(u_{0}\right), u_{0}\right\rangle$ by (A4) and $\left\langle B^{\prime}\left(u_{j}\right), u_{j}\right\rangle \rightarrow\left\langle B^{\prime}\left(u_{0}\right), u_{0}\right\rangle$ by (B2). Then from (3.13) follows:

$$
\begin{gathered}
D^{+} \sigma\left(t_{0}\right) \leq \sup \left\{\rho \in \Lambda_{t_{0}}^{(\rho)}\right\}, \\
D_{-} \sigma\left(t_{0}\right) \geq \inf \left\{\rho \in \Lambda_{t_{0}}^{(\rho)}\right\} .
\end{gathered}
$$

3. Let us combine (3.7) and (3.14). Then

$$
\sup \left\{\rho \in \Lambda_{t_{0}}^{(\rho)}\right\} \leq D_{+} \sigma\left(t_{0}\right) \leq D^{+} \sigma\left(t_{0}\right) \leq \sup \left\{\rho \in \Lambda_{t_{0}}^{(\rho)}\right\}
$$


i.e., $\sigma_{+}^{\prime}\left(t_{0}\right)$ exists and satisfies (3.1). Similarly, (3.2) follows from (3.8) and (3.15).

4. Continua of solutions. Range of solvability. In this section we assume the conditions of Theorem 3.1.

Proposition 4.1. Assume that for every $t \in\left(s_{1}, s_{2}\right) \subset(0, \infty)$ the set $\Lambda_{t}^{(u)}$ consists of a single element $u_{t}$. Then the map $t \rightarrow u_{t}$ is weakly continuous on $\left(s_{1}, s_{2}\right)$.

Proof. Let $t_{0} \rightarrow t_{0} \in\left(s_{1}, s_{2}\right)$. Then $\overline{\left\{t_{j}\right\}}$ is compact in $(0, \infty)$ and by Lemma $2.3 u_{t}$, has a (renamed) weakly convergent subsequence $u_{t_{j}} \stackrel{w}{\longrightarrow} u_{0} \in \Lambda_{t_{0}}^{(u)}$. Since $\Lambda_{t_{0}}^{(u)}=\left\{u_{t_{0}}\right\}$, the original sequence $u_{t_{j}}$ must be weakly convergent to the same element $u_{t_{0}}$.

COROllary 4.2. Under conditions of Proposition 4.1 the problem

$$
\rho A^{\prime}(u)=B^{\prime}(u)
$$

possesses a weakly continuous family of eigenfunctions $t \rightarrow u_{t}$ corresponding to eigenvalues $\rho_{t}=\left\langle B^{\prime}\left(u_{t}\right), u_{t}\right\rangle /\left\langle A^{\prime}\left(u_{t}\right), u_{t}\right\rangle$ continuous in $t \in\left(s_{1}, s_{2}\right)$. The function (2.3) has a continuous derivative on $\left(s_{1}, s_{2}\right)$ and $\rho_{t}=\sigma^{\prime}(t)$.

The proof follows from Theorems 3.1 and 3.2.

Proposition 4.3. Let $h: X^{2} \rightarrow X$ be a map, such that

(Ah) $A(h(u, v))>\max \{A(u), A(v)\}$ for $u \neq v$.

(Bh) $B(h(u, v)) \geq \min \{B(u), B(v)\}$ for $u, v \in X$.

Then the assertions of Proposition 4.1 and Corollary 4.2 hold for any $t>0$.

Proof. Let $u, v \in \Lambda_{t}^{(u)} u \neq v$. Then by (Ah), $h(u, v) \in \omega_{t^{\prime}}, t^{\prime}<t$, but by $(\mathrm{Bh}) B(h(u, v)) \geq B(u)=\sigma(t)$. This contradicts Lemma 2.1. Thus the conditions of Lemma 4.1 are satisfied at any $t>0$.

A simple example when (Ah), (Bh) are satisfied, can be provided by

Corollary 4.4. Let $A$ be strictly convex and $B$ be concave on $X$. Then the assertions of Proposition 4.1 and Corollary 4.2 hold for $t \in(0, \infty)$.

Proof. Take $h(u, v)=\lambda u+(1-\lambda) v, \lambda \in(0,1)$. 
If $\sigma \in C_{\text {loc }}^{1}(0, \infty)$, then (4.1) is solvable (with $u \neq 0$ ) for any $\rho \in I(A, B)$, where

$$
I(A, B)=\left(\inf _{t} \sigma_{-}^{\prime}(t), \sup _{t} \sigma_{+}^{\prime}(t)\right) .
$$

However, $\sigma$ does not generally have a continuous derivative (cf. [4]). Thus we wish to answer the question, for what subsets of $I(a, b)$ does (4.1) still have a non-zero solution.

Proposition 4.5. Let the function $\rho t-\sigma(t)$ have a local minimum on $(0, \infty)$. Then $(4.1)$ has a non-zero solution.

Proof. Let $\rho t-\sigma(t)$ have a local minimum at $t_{0}>0$. Then $\sigma_{-}^{\prime}\left(t_{0}\right) \geq \rho \geq \sigma_{+}^{\prime}\left(t_{0}\right)$. By Theorem 3.1, $\sigma_{-}^{\prime}\left(t_{0}\right) \leq \sigma_{+}^{\prime}\left(t_{0}\right)$. Thus, $\sigma$ is differentiable at $t_{0}$ and $\sigma^{\prime}\left(t_{0}\right)=\rho$. Then by Theorem 3.2, (4.1) has a solution with $A(u)=t_{0}$.

In order to get a more extensive result we use a mountain pass theorem from [5].

Theorem 4.6. Let $G \in C^{1}(X \rightarrow \mathbf{R})$ and let $u_{0} \in X \backslash\{0\}$. Let $N \subset X$ be an open bounded set, such that $0 \in N$ but $u_{0} \notin \bar{N}$. Assume that

$$
\begin{array}{cc}
G(u) \geq 0, & u \in \partial N, \\
G(0) \leq 0, & G\left(u_{0}\right) \leq 0 .
\end{array}
$$

Then there is a sequence $u_{k} \in X$ and $\gamma \geq 0$ such that

$$
\begin{gathered}
G\left(u_{k}\right) \rightarrow \gamma, \\
\left\|G^{\prime}\left(u_{k}\right)\right\|_{X^{*}}\left\|u_{k}\right\|_{X} \rightarrow 0 .
\end{gathered}
$$

Let now

and let

$$
\begin{gathered}
A_{\theta}=\theta\left\langle A^{\prime}(u), u\right\rangle-A(u), \\
B_{\theta}=\theta\left\langle B^{\prime}(u), u\right\rangle-B(u), \\
\Phi=\left\{\theta \in \mathbf{R}:\left|A_{\theta}\right| \text { is coercive }\right\}
\end{gathered}
$$

(4.10) $Q(\theta)$ be set of limit points for $B_{\theta}(u) / A_{\theta}(u)$

$$
\text { when }\|u\| \rightarrow \infty, \theta \in \Phi \text {. }
$$

Set now

$$
Q_{*}=\bigcap_{\theta \in \Phi} Q(\theta)
$$


Theorem 4.7. Assume, in addition to conditions of Theorem 3.1, that:

(ABw) $A^{\prime}, B^{\prime}$ are continuous from $X$ to $X^{*}$ with regard to respective weak topologies.

Then for every $\rho \in I(A, B) \backslash Q_{*}$ the equation (4.1) has a nonzero solution.

Proof. 1. Let $\rho \in I(A, B) \backslash Q_{*}$, i.e., $\rho$ is a slope of a secant to the graph of $\sigma(t)$, the functional $\left|A_{\theta}\right|$ is coercive for some $\theta$ and $\rho$ is not a limit point of $B_{\theta} / A_{\theta}$ at infinity. If $\rho t-\sigma(t)$ has a local minimum at $t \neq 0$, then $\rho$ is an eigenvalue (Proposition 4.5). Thus we would consider the case when $\rho t-\sigma(t)$ has no local minimum. If $\rho=\sigma_{+}^{\prime}\left(t_{0}\right)$ or $\rho=\sigma_{-}^{\prime}\left(t_{0}\right)$ for some $t_{0}>\theta$, then it is an eigenvalue by Theorem 3.2. The remaining case is: for some $t_{0}>0, \sigma^{\prime}\left(t_{0}\right)<\rho \sigma_{+}^{\prime}\left(t_{0}\right)$. This implies that $t_{0}$ is a point of local maximum of $\rho t-\sigma(t)$. Since we assume that $\rho t-\sigma(t)$ has no local minimum, $t_{0}$ is a point of strict global maximum. In particular, $\delta:=\rho t_{0}-\sigma\left(t_{0}\right)>0-\sigma(0)=0$, and there exists $t_{1}>t_{0}$, such that $\delta_{1}:=\rho t_{1}-\sigma\left(t_{1}\right)<\delta$.

2. Let

$$
G(u)=\rho A(u)-B(u)-\delta_{1} .
$$

Then all the conditions of Theorem 4.6 are fulfilled with $N=\{u \in$ $\left.X, A(u) \leq t_{0}\right\}$ and $u_{0} \in S_{t_{1}}$. Let $u_{k} \in X, \gamma \geq 0$ satisfy

$$
\begin{gathered}
\rho A\left(u_{k}\right)-B\left(u_{k}\right) \rightarrow \delta_{1}+\gamma \geq \delta_{1}, \\
\left\|\rho A^{\prime}\left(u_{k}\right)-B^{\prime}\left(u_{k}\right)\right\|_{X^{*}}\left\|u_{k}\right\|_{X} \rightarrow 0 .
\end{gathered}
$$

Then

$$
\rho\left\langle A^{\prime}\left(u_{k}\right), u_{k}\right\rangle-\left\langle B^{\prime}\left(u_{k}\right), u_{k}\right\rangle \rightarrow 0
$$

and, consequently,

$$
\rho A_{\theta}\left(u_{k}\right)-B_{\theta}\left(u_{k}\right) \rightarrow-\gamma-\delta .
$$

If $\left\|u_{k}\right\| \rightarrow \infty$, then $\left|A_{\theta}\left(u_{k}\right)\right| \rightarrow \infty, \rho=\lim B_{\theta}\left(u_{k}\right) / A_{\theta}\left(u_{k}\right)$, which contradicts the assumptions. Thus the sequence $u_{k}$ is bounded in norm. Let now $u_{k}$ be a renamed weakly convergent sequence, and $u_{0}=w$-lim $u_{k}$. Then by (ABw) from (4.14) follows:

$$
\rho A^{\prime}\left(u_{0}\right)=B^{\prime}\left(u_{0}\right) \text {. }
$$

Moreover, by (B3), (4.14), (4.17)

$$
\begin{aligned}
\lim \rho\left\langle A^{\prime}\left(u_{k}\right), u_{k}\right\rangle & =\lim \left\langle B^{\prime}\left(u_{k}\right), u_{k}\right\rangle=\left\langle B^{\prime}\left(u_{0}\right), u_{0}\right\rangle \\
& =\rho\left\langle A^{\prime}\left(u_{0}\right), u_{0}\right\rangle .
\end{aligned}
$$


Then by (A4)

$$
\lim A\left(u_{k}\right)=A\left(u_{0}\right)
$$

Thus by (4.13), (B2),

$$
0<\delta_{1}+\gamma=\lim \rho A\left(u_{k}\right)-B\left(u_{k}\right)=\rho A\left(u_{0}\right)-B\left(u_{0}\right)
$$

which proves that $u_{0} \neq 0$.

Corollary 4.8. Let $\rho_{*}=\inf _{\theta \in \Phi} \lim \sup _{\|u\| \rightarrow \infty} B_{\theta}(u) / A_{\theta}(u)$. Then (4.1) has a non-zero solution for

$$
\rho \in I(A, B) \cap\left(\rho_{*}, \infty\right) \text {. }
$$

5. Applications to quasilinear elliptic problems. Let $\Omega \subset \mathbf{R}^{n}$ be an open bounded set $X=W_{0}^{l, p}(\Omega)^{k}, p>1, l \in \mathbf{N}, k \in \mathbf{N}$. Let $\nu(l)$ be the number of multi-indices of length not exceeding $l$. Assume that $\mathscr{A}\left(x,\left\{y_{\alpha}\right\}_{|\alpha| \leq l}\right)$ and $\mathscr{B}\left(x,\left\{y_{\alpha}\right\}_{|\alpha| \leq l-1}\right)$ are $C^{1}$ real valued functions of $\left\{y_{\alpha}\right\}$ whose derivatives are Carathéodory functions of $\left(x,\left\{y_{\alpha}\right\}\right)$. Without loss of generality we assume that

$$
\mathscr{A}(x, 0)=0, \quad \mathscr{B}(x, 0)=0 .
$$

We require for the function $\mathscr{A}$ the following ellipticity condition:

$$
\mathscr{A}(x, \cdot) \text { is convex for almost every } x \in \Omega
$$

and the following coercivity condition:

$$
\left.\frac{d}{d t} \mathscr{A}\left(x,\left\{t y_{\alpha}\right\}\right)\right|_{t=1} \geq c \sum_{|\alpha|=l}\left|y_{\alpha}\right|^{p}, \quad c>0, x \in \Omega .
$$

We also require the following upper bounds for $\mathscr{A}^{\prime}$ and $\mathscr{B}^{\prime}$ :

$$
\left|\mathscr{A}_{y_{\alpha}^{\prime}}^{\prime}\left(x,\left\{y_{\alpha}\right\}\right)\right| \leq c\left(1+\sum_{|\alpha| \leq l}\left|y_{\alpha}\right|^{p-1}\right), \quad c>0
$$

$$
\begin{aligned}
\left|\mathscr{B}_{y_{\alpha}}^{\prime}\left(x,\left\{y_{\alpha}\right\}\right)\right| \leq & C \sum_{\substack{|\beta| \leq l-1 \\
|\beta| \geq l-n / p}}\left|y_{\beta}\right|^{q_{\alpha \beta}} \\
& +V_{\alpha}\left(\left\{y_{\beta}\right\}_{|\beta|<l-n / p}\right)+W_{\alpha}(x),
\end{aligned}
$$

where

$$
C>0
$$$$
W_{\alpha} \in L^{r}, r_{\alpha}=1 \text { if } l-|\alpha|>n / p \text {, }
$$$$
r_{\alpha}>p n /(p n-n-p(l-|\alpha|)) \text { if } l-|\alpha| \leq n / p \text {, }
$$

$V_{\alpha}$ is a continuous function, 


$$
\begin{aligned}
& q_{\alpha \beta}>\frac{p n}{n-p(l-|\beta|)} \text { if } l-|\alpha|>n p, \\
& q_{\alpha \beta}<\frac{p n-n+p(l-|\alpha|)}{n-p(l-|\beta|)}, \quad \text { if } l-|\alpha| \leq n / p .
\end{aligned}
$$

Proposition 5.1. Assume (5.2)-(5.6). Then the functionals

$$
\begin{aligned}
& A(u)=\int_{\Omega} \mathscr{A}\left(x,\left\{\partial^{\alpha} u\right\}\right) d x, \\
& B(u)=\int_{\Omega} \mathscr{B}\left(x,\left\{\partial^{\alpha} u\right\}\right) d x
\end{aligned}
$$

satisfy (A1-A4), (B2), (B3), (ABw) on $X=W_{0}^{l, p}(\Omega)^{k}$.

Proof. The verification of continuity and differentiability properties is standard and based on compactness in the Sobolev embedding theorem. We wish to make remarks on only a few details.

1. Relation (5.3) implies (A3) and also (A1), since it immediately gives

$$
\frac{d}{d t} \mathscr{A}\left(x,\left\{t y_{\alpha}\right\}\right) \geq c t^{p-1} \sum_{|\alpha|=l}\left|y_{\alpha}\right|^{p}, \quad t>0 .
$$

2. Relation (5.2) implies that the set (2.2) is convex. Thus it is weakly closed and $A$ is lower semicontinuous.

3. Due to (5.9), (5.4) weak convergence of a sequence $u_{j}$ together with convergence of either $A\left(u_{j}\right)$ or of $\left\langle A^{\prime}\left(u_{j}\right), u_{j}\right\rangle$ is equivalent to convergence in norm.

To verify the condition (B1) in most of the applications it suffices to prove that $B^{\prime}(u) \neq 0$ unless $u=0$ and that $u=0$ is not a point of maximum. Two particular cases are given below.

\section{LEMMA 5.2. Let}

$$
\sum_{\alpha} \mathscr{B}_{y_{\alpha}}^{\prime}\left(x,\left\{y_{\alpha}\right\}\right) y_{\alpha}>0 \text { for }\left\{y_{\alpha}\right\} \neq 0
$$

Then (B1) holds.

Proof. From (5.10) it follows that $\left\langle B^{\prime}(u), u\right\rangle>0$ unless $u=0$. The point $u=0$ is not a point of maximum, but rather of minimum, since for every $u \in X \backslash\{0\}, B$ increases along the line $t \rightarrow t u$, $t>0$. 
LEMMA 5.3. Let $k=1, b\left(x,\left\{y_{\alpha}\right\}\right)=F\left(y_{0}\right)$ and assume that with some $\varepsilon>0$,

$$
\begin{array}{ll}
F^{\prime}\left(y_{0}\right)>0 & \text { for } y_{0} \in(0, \varepsilon) \text { and } \\
F^{\prime}\left(y_{0}\right) \neq 0 & \text { for } y_{0} \in(-\varepsilon, 0) .
\end{array}
$$

Then (B1) holds.

Proof. Let $u$ be the point of maximum of $b$ and let $\bar{u}$ be a decreasing spherical rearrangement for $u$. Then $\bar{u}$ is a $W_{0}^{l, \rho}$-function dependent on the radial variable only. Therefore $\bar{u}$ is continuous away from the origin, the range of $\bar{u}$ is a closed interval $I$ containing zero. Moreover, the range of $u$ is dense in $I$, for if $(s, t)$ is not in the range of $u,(s, t)$ is not in the range of $\bar{u}$. If $u$ is a point of maximum for $B$, then $F^{\prime}=0$ on the range of $u$, and since $F^{\prime}$ is continuous $F^{\prime}=0$ on $I$. By $(5.11)$, therefore, $I \cap(-\varepsilon, \varepsilon) \subset\{0\}$. However, $u=0$ is not a maximum: one can perturb 0 by a function $v \geq 0$ of an arbitrarily small norm so that $B(v)>B(0)=0$.

The following statement is now an immediate corollary of Theorems 3.1, 3.2.

TheOREM 5.4. Assume (5.1-5.6) and (B1). Then for every $t>0$ there exists a semistrong solution $u_{t}^{ \pm} \in W_{0}^{l, p}(\Omega)^{k}$ satisfying the respective equations:

$$
\begin{aligned}
\sigma_{ \pm}^{\prime}(t) & \sum_{|\alpha| \leq l}(-1)^{|\alpha|} \partial^{\alpha} \mathscr{A}_{y_{\alpha}^{\prime}}^{\prime}\left(x,\left\{\partial^{\gamma} u(x)\right\}\right) \\
& =\sum_{|\beta| \leq l-1}(-1)^{|\beta|} \partial^{\beta} \mathscr{B}_{y_{\beta}}^{\prime}\left(x,\left\{\partial^{\gamma} u(x)\right\}\right) .
\end{aligned}
$$

Moreover,

$$
A\left(u_{t}^{ \pm}\right)=t
$$

and the function $\sigma$ is given by

$$
\sigma(t)=\sup _{A(u)=t} B(u)
$$

We now will look for realization of conditions $(\mathrm{Ah}),(\mathrm{Bh})$ to get continuous curves of eigenfunctions. 
THEOREM 5.5. Assume (5.1-5.6) and (B1). Let $\mathscr{B}(x, \cdot)$ be concave for a.e. $x \in \Omega$. Then there is a continuous family $t \rightarrow\left(\rho_{t}, u_{t}\right) \in$ $(0, \infty) \times W_{0}^{l, p}(\Omega)^{k}$, such that

$$
\begin{aligned}
& \rho_{t} \sum_{|\alpha| \leq l}(-1)^{|\alpha|} \partial^{\alpha} \mathscr{A}_{y_{\alpha}}^{\prime}\left(x,\left\{\partial^{\gamma} u_{t}(x)\right\}\right) \\
& \quad=\sum_{|\alpha| \leq l-1}(-1)^{|\alpha|} \partial^{\alpha} \mathscr{B}_{y_{\alpha}}^{\prime}\left(x,\left\{\partial^{\gamma} u_{t}(x)\right\}\right) .
\end{aligned}
$$

Proof. Apply Corollary 4.4. Then $u_{t}$ satisfies (5.15) and $t \rightarrow u_{t}$ is weakly continuous. However, by (5.4), (5.9) convergence of $t_{j}=$ $A\left(u_{t_{j}}\right)$ together with weak convergence of $u_{t_{j}}$ is equivalent to convergence in norm and the family $\left(\rho_{t}, u_{t}\right)$ is continuous.

THEOREM 5.6. Let $\mathscr{A}\left(x,\left\{\partial^{\alpha} u\right\}\right)=|\nabla u|^{p}, \mathscr{B}\left(x,\left\{y_{\alpha}\right\}\right)=F\left(y_{0}\right)$, $k=1$. Assume also that $F \in C^{1}$,

$$
F^{\prime}(s)>0 \text { for } s>0 \text { and } F^{\prime}(s)=0 \text { for } s \leq 0,
$$

and that the map

$$
s \rightarrow F\left(s^{1 / p}\right) \text { is concave for } s>0 .
$$

Then there is a continuous family $t \rightarrow\left(\rho_{t}, u_{t}\right)$ satisfying

$$
-\rho_{t} \operatorname{div}\left(\left|\nabla u_{t}\right|^{p-2} \nabla u_{t}\right)=F^{\prime}\left(u_{t}\right), \quad u_{t} \geq 0 .
$$

Proof. Let

$$
h_{\theta}\left(u_{1}, u_{2}\right)=\left(\theta\left|u_{1}\right|^{p}+(1-\theta)\left|u_{2}\right|^{p}\right)^{1 / p}, \quad 0 \in(0,1) .
$$

Then, applying Hölder inequality with $\frac{1}{p}+\frac{1}{p^{\gamma}}=1$,

$$
\begin{aligned}
& \left|\nabla\left(\theta\left|u_{1}\right|^{p}+(1-\theta)\left|u_{2}\right|^{p}\right)^{1 / p}\right|^{p} \\
& \quad=\left|\frac{1}{p} \frac{\theta p\left|u_{1}\right|^{p-1} \nabla\left|u_{2}\right|+(1-\theta) p\left|u_{2}\right|^{p-1} \nabla\left|u_{2}\right|}{\left(\theta\left|u_{1}\right|^{p}+(1-\theta)\left|u_{2}\right|^{p}\right)^{1 / p^{\prime}}}\right|^{p} \\
& \quad=\left|\frac{\theta^{1 / p+1 / p^{\prime}}\left|u_{1}\right|^{p / p^{\prime}} \nabla\left|u_{1}\right|+(1-\theta)^{1 / p+1 / p^{\prime}}\left|u_{2}\right|^{p / p^{\prime}} \nabla\left|u_{2}\right|}{\left(\theta\left|u_{1}\right|^{p}+(1-\theta)\left|u_{2}\right|^{p}\right)^{1 / p^{\prime}}}\right|^{p} \\
& \quad \leq \theta|\nabla| u_{1}||^{p}+(1-\theta)|\nabla| u_{2}||^{p} \\
& \quad=\theta\left|\nabla u_{1}\right|^{p}+(1-\theta)\left|\nabla u_{2}\right|^{p} .
\end{aligned}
$$

The relation (5.20) makes sense a.e. when $u_{1}, u_{2} \in C_{0}^{1}(\Omega)$ and the equality holds only if $u_{1}=u_{2}$ or one of them vanishes. Then the following will be true on $W_{0}^{1, p}(\Omega)$ :

$$
A\left(h_{\theta}\left(u_{1}, u_{2}\right)\right) \leq \theta A\left(u_{1}\right)+(1-\theta) A\left(u_{2}\right)
$$


with the same remark on equality. By (5.16), (5.17),

$$
B\left(h_{\theta}\left(u_{1}, u_{2}\right)\right) \leq \theta B\left(u_{1}\right)+(1-\theta) B\left(u_{2}\right) \text {. }
$$

Thus (Ah), (Bh) are satisfied and the assertion follows from Theorem 5.11. One has only to note that $t_{j} \rightarrow t_{0}$ and $u_{t_{i}} \stackrel{w}{\longrightarrow} u_{t_{0}}$ implies $u_{t_{j}} \rightarrow t_{0}$.

Now we wish to find some realizations of Theorem 4.7.

LeMmA 5.7. Assume (5.1-5.6) and (B1). Let

$$
\mathscr{B}_{y_{\alpha}}^{\prime}\left(x,\left\{y_{\alpha}\right\}\right)=o\left(\sum_{|\alpha| \leq l-1}\left|y_{\alpha}\right|^{p-1}\right) \text { uniformly in } \Omega \text {. }
$$

Then

$$
Q_{*} \subset\{0\} .
$$

Proof. By (5.3), (5.4), $A_{\theta} \geq\|u\|^{p}$ with $\theta$ sufficiently large. By (5.23), $B_{\theta}(u)=o\left(\|u\|^{p}\right)$. Thus $Q_{*} \subset\{0\}$.

TheOREM 5.8. Assume (5.1-5.6), (B1) and (5.23). Then for every

$$
\rho \in\left(0, \sup _{t} \sigma_{+}^{\prime}(t)\right)
$$

there is a non-zero solution of

$$
\begin{aligned}
& \rho \sum_{|\alpha| \leq l}(-1)^{|\alpha|} \partial^{\alpha} \mathscr{A}\left(x,\left\{\partial^{\gamma} u(x)\right\}\right) \\
& \quad=\sum_{|\beta| \leq l-1}(-1)^{|\beta|} \partial^{\beta} \mathscr{B}\left(x,\left\{\partial^{\gamma} u(x)\right\}\right) .
\end{aligned}
$$

Moreover,

$$
\sup _{t} \sigma_{+}^{\prime}(t) \geq \sup _{u \neq 0} B(u) / A(u)
$$

Proof. By Theorem 4.7, (5.26) is solvable for $\rho \in I(a, b)$. The lower bound in $I(a, b)$ is less than $\sigma(t) / t$ which goes to zero when $t$ tends to $\infty$. The upper bound of $I(a, b)$ is greater or equal to any given slope of a secant line to the graph of $\sigma$, e.g. $\sigma(t) / t$, which implies (5.27).

TheOREM 5.9. Assume that $k=1$,

$$
\mathscr{B}\left(x,\left\{y_{\alpha}\right\}\right)=F\left(y_{0}\right), \quad \mathscr{A}\left(x,\left\{\partial_{x}^{\alpha} u\right\}\right)=|\nabla u|^{p} .
$$


Let $F \in C^{1}$ and if $n \geq p$, let $F^{\prime}(s)=o\left(|s|^{q-1}\right), q<p n /(n-p)$. Assume that for some $\varepsilon>0$

$F^{\prime}(s) / s^{p-1+\varepsilon}$ is an increasing function

in a neighbourhood of $+\infty$,

$$
F^{\prime}(s)=o\left(|s|^{p-1}\right) \text { as } s \rightarrow-\infty .
$$

Then for every

$$
\rho \in\left(\inf _{t} \sigma_{-}^{\prime}(t), \infty\right)
$$

there is a solution $u \neq 0$ for

$$
-\rho p \operatorname{div}\left(|\nabla u|^{p-2} \nabla u\right)=F^{\prime}(u) .
$$

Proof. Note that $\mathscr{A}, \mathscr{B}$ satisfy $(5.1-5.6)$ and B1.

From $(5.28)$ it follows that $\mathscr{B}_{\theta}(u) \leq o\left(\|u\|^{p}\right)$ for $\theta>1 /(p+\varepsilon)$. Thus $Q_{*} \cap(0, \infty)=\varnothing$, and one can apply Theorem 4.7. By (5.8) $\sup \sigma_{+}^{\prime}(t) \geq \sup _{t} \sigma(t) / t=\infty$.

As a general realization of 4.7 we state:

TheOrem 5.10. Assume (5.1-5.6) and (B1). Then (5.26) has a nonzero solution $u$ for

$$
\rho \in\left(\inf \sigma_{-}^{\prime}(t), \sup \sigma_{+}^{\prime}(t)\right) \backslash Q_{*} .
$$

6. Examples. The following examples illustrate the solvability results of this paper.

EXAMPLE 6.1. Let $\Omega \subset \mathbf{R}^{n}$ be an open bounded set. Consider

$$
\left\{\begin{array}{l}
-\rho \operatorname{div}\left(|\nabla|^{p-2} \nabla u\right)=u^{\alpha}+u^{\beta}, \quad u \geq 0, p>1, \\
\left.u\right|_{\partial \Omega}=0 .
\end{array}\right.
$$

Case 1. $0<\alpha<\beta<p-1$. Then by Theorem 5.6 there is a continuous family $\left(\rho_{t}, u_{t}\right)$ of eigenfunctions. By Theorem 5.4 , the range of eigenvalues $\rho$ is $I(A, B)$ which is here $(0, \infty)$.

Case 2. $0<\alpha<p-1<\beta$. If $n>p$ assume also $\beta<n p /(n-p)$ -1 . Then (6.1) has a solution for every $\rho \in\left(\rho_{0}, \infty\right), \rho_{0}>0$ and

$$
\begin{aligned}
\rho_{0} \leq \inf _{t} \sup _{\|u\|_{1, p}=1} t^{-1} \int_{\Omega}\left(\frac{p}{\alpha+1} u^{\alpha+1} t^{(\alpha+1) / p}\right. \\
\left.+\frac{p}{\beta+1} u^{\beta+1} t^{(\beta+1) / p}\right) d x .
\end{aligned}
$$


Solvability for $\rho \in I(A, B)$ is provided by Theorem 5.9.

Case 3. $p-1<\alpha<\beta$ and if $n>p, \beta<n p /(n-p)-1$. Similarly, (6.1) is solvable with $\rho \in(0, \infty)$ by Theorem 5.9.

Case 4. $0<\alpha<\beta=p-1$. The argument is like in Case 1 , only $I(A, B)=\left(0, \rho_{0}\right)$,

$$
\rho_{0}=\sup _{\|u\|_{l, p}=1} \frac{1}{p} \int|u|^{p}
$$

Case 5. $0<\alpha=p-1<\beta$, if $n>p, \beta<n p /(n-p)-1$. The argument follows one of Case 3 , but $I(a, b)=\left(\rho_{0}, \infty\right)$, where $\rho_{0}$ is like in (6.3).

EXAMPLE 6.2. Let $\Omega \subset \mathbf{R}^{n}, n<6$, be an open bounded domain. Consider a system:

$$
\left\{\begin{array}{l}
-3 \rho \operatorname{div}\left(|\nabla u|+\frac{1}{2}|\nabla v|^{3 / 2}|\nabla u|^{-1 / 2}\right) \nabla u=5 u^{4}, \\
-3 \rho \operatorname{div}\left(|\nabla v|+\frac{1}{2}|\nabla u|^{3 / 2}|\nabla v|^{-1 / 2}\right) \nabla v=4 v^{3}, \quad u,\left.v\right|_{\partial \Omega}=0 .
\end{array}\right.
$$

This system corresponds to

$$
\begin{aligned}
& A(u, v)=\int_{\Omega}\left(|\nabla u|^{3}+|\nabla v|^{3}+|\nabla u|^{3 / 2}|\nabla v|^{3 / 2}\right) d x, \\
& B(u, v)=\int_{\Omega}\left(u^{5}+v^{4}\right) d x .
\end{aligned}
$$

By Theorem 5.9, it is solvable for $\rho \in I(A, B)$ and $I(A, B)=(0, \infty)$.

\section{REFERENCES}

[1] M. S. Berger, Nonlinearity and Functional Analysis, Academic Press 1977.

[2] F. E. Browder, Infinite dimensional manifolds and non-linear elliptic eigenvalue problems, Ann. of Math., 82 (1965), 459-477.

[3] M. Schechter and K. Tintarev, Spherical maxima in Hilbert space and semilinear elliptic problems, Differential Equations, 3(5) (1990), 889-899.

[4] __, Eigenvalues for semilinear boundary value problem, Arch. Rational Mech. Anal., 113 (1991), 197-208.

[5] M. Schechter, $A$ variation of the mountain pass lemma and applications, J. London Math. Soc., (to appear).

Received July 26, 1990 and in revised form May 13, 1991. 


\section{PACIFIC JOURNAL OF MATHEMATICS EDITORS}

V. S. VARADARAJAN

(Managing Editor)

University of California

Los Angeles, CA 90024-1555-05

Herbert Clemens

University of Utah

Salt Lake City, UT 84112

F. Michael Christ

University of California

Los Angeles, CA 90024-1555

THOMAS ENRIGHT

University of California, San Diego

La Jolla, CA 92093

\section{C. MOORE}

University of California

Berkeley, CA 94720

MARTIN SCHARLEMANN

University of California

Santa Barbara, CA 93106

HAROLD STARK

University of California, San Diego

La Jolla, CA 92093

STEVEN KERCKHOFF

Stanford University

Stanford, CA 94305

ASSOCIATE EDITORS

R. ARENS

E. F. BECKENBACH (1906-1982)

B. H. NeumanN

F. WOLF

(1904-1989)

K. YosHIDA

SUPPORTING INSTITUTIONS

UNIVERSITY OF ARIZONA

UNIVERSITY OF OREGON

UNIVERSITY OF BRITISH COLUMBIA

UNIVERSITY OF SOUTHERN CALIFORNIA

CALIFORNIA INSTITUTE OF TECHNOLOGY

UNIVERSITY OF CALIFORNIA

MONTANA STATE UNIVERSITY

STANFORD UNIVERSITY

UNIVERSITY OF NEVADA, RENO

UNIVERSITY OF HAWAII

NEW MEXICO STATE UNIVERSITY

OREGON STATE UNIVERSITY

UNIVERSITY OF UTAH

WASHINGTON STATE UNIVERSITY

UNIVERSITY OF WASHINGTON 


\section{Pacific Journal of Mathematics}

\section{Vol. 153, No. $1 \quad$ March, 1992}

Patrick Robert Ahern and Carmen Cascante, Exceptional sets for Poisson integrals of potentials on the unit sphere in $\mathbf{C}^{n}, p \leq 1 \ldots \ldots \ldots \ldots \ldots$

David Peter Blecher, The standard dual of an operator space ........... 15

Patrick Gilmer, Real algebraic curves and link cobordism .............. 31

Simon M. Goberstein, On orthodox semigroups determined by their

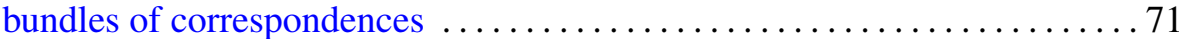

John Kalliongis and Darryl John McCullough, Homeotopy groups of irreducible 3-manifolds which may contain two-sided projective planes .......................................... 85

Yuji Konishi, Masaru Nagisa and Yasuo Watatani, Some remarks on actions of compact matrix quantum groups on $C^{*}$-algebras $\ldots \ldots \ldots \ldots 119$

Guojun Liao and Luen-Fai Tam, On the heat equation for harmonic maps from noncompact manifolds ........................... 129

John Marafino, Boundary behavior of a conformal mapping . .......... 147

Ji Min, A remark on the symmetry of solutions to nonlinear elliptic

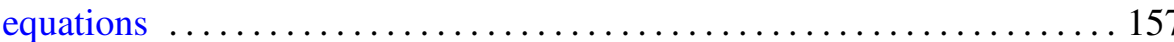

Paul Nevai and Walter Van Assche, Compact perturbations of orthogonal

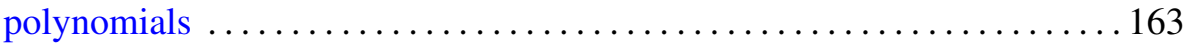

Kyril Tintarev, Level set maxima and quasilinear elliptic problems ....... 185 